Chairs: Håkon Hope, Elisa Nauha

\section{MS17-01 Understanding the polymorphic phase transitions of linear amino acids using in situ characterisation}

Mireille Smets ${ }^{1}$, Sander Brugman ${ }^{1}$, Ernst van Eck ${ }^{1}$, Joost van den Ende $^{1}$, Hugo Meekes ${ }^{1}$, Elias Vlieg ${ }^{1}$, Herma Cuppen ${ }^{1}$

1. Institute for Molecules and Materials, Radboud University, Heyendaalseweg 135, 6525 AJ Nijmegen, The Netherlands

\section{email:m.smets@science.ru.nl}

Polymorphism is a common phenomenon for molecular crystals. Here we aim to understand the mechanisms involved in transitions between polymorphic forms in the solid state. Ultimately, the understanding will allow for the control of polymorphic forms by promoting or inhibiting transitions, which is important for prolonging the shelf life of polymorphic forms of active pharmaceutical ingredients (API). The established classification of phase transitions cannot explain several phenomena in molecular systems, such as cooperative motion ${ }^{1}$. This mechanism has been inferred to explain thermosalient behaviour ${ }^{2}$. I will present the in-situ characterisation of the reversible $\alpha-\beta$ and $\alpha-\gamma$ phase transitions of DL-norleucine (NLE) and the $\alpha-\beta$ transition of DL-methionine (MET). Although all polymorphs involved have been reported in the literature, little is known about the transition between the structures. I will show that the transition behaviour in these linear amino acids can be explained by cooperative motion.

NLE is an amino acid with three known polymorphic forms, which consist of zwitterionic H-bonded bilayers. The three monoclinic structures differ in the stacking of the bilayers $(\beta \leftrightarrow \alpha, \alpha \leftrightarrow \gamma)$ and in the conformation of the molecules $(\alpha \leftrightarrow \gamma)^{3}$. MET has two known polymorphic forms $(\beta \& \alpha)$ with different bilayer stacking and different conformations 4

We studied the transitions using complementary in situ techniques (DSC, SCXRD, solid-state NMR, polarisation microscopy) to get a full picture of the phase transitions. Especially solid-state NMR proved to be a powerful technique, due to its sensitivity to changes in the environment of atoms. The $\alpha-\gamma$ phase transition of NLE behaves as a typical first order phase transition with the nucleation and growth mechanism. The $\alpha-\beta$ phase transition of NLE is difficult to observe and occurs very irregularly. It shows coexistence and a large dependence on crystal quality and defect density that suggests cooperative motion. ${ }^{5}$ This is in agreement with molecular dynamics simulations. $^{6}$ The $\alpha-\beta$ transition of MET shows intermediate behaviour and is consistent with kinetic hindrance.
1.Brandel et al, Chem. Mat. 27,6360-6373 (2015).

2.Naumov et al, Chem. Rev. 115,12440-12490 (2015).

3. Coles et al, Cryst. Growth \& Des. 9,4610-4612 (2009). 4.Görbitz et al, Acta Cryst E. 70,337-340 (2014), Acta Cryst E. 71,0398-o399 (2015). 5.Smets et al, Cryst. Growth Des. 15,5157-5167 (2015). 6.Van den Ende, Smets, et al, Faraday Discuss. 179,421-436 (2015).

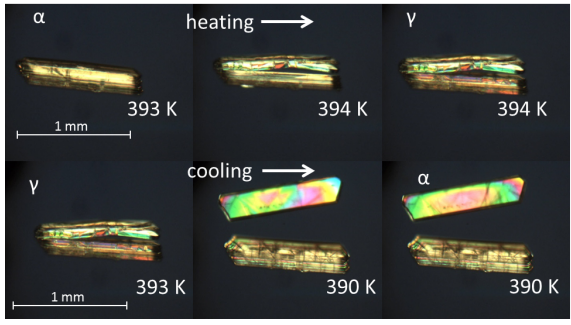

Figure 1. Thermal stage polarisation microscopy snapshots of a single crystal of DL-norleucine at different temperatures during the $\alpha \leftrightarrow \gamma$ transition. During the transition, the crystal delaminates because of the anisotropic lattice expansion.

Keywords: solid-state phase transitions, polymorphism, amino acids, in situ characterisation 